\title{
A Hybrid Optimization Algorithm for Enhanced Web Services with QoS Measures in Cloud Computing.
}

\section{Thenmozhi ( $\nabla$ Thenmozhiphd08@yahoo.com )}

MGR College https://orcid.org/0000-0001-8465-5095

\section{N. Chandrakala}

SSM College of Arts and Science

\section{Research Article}

Keywords: Hybrid Invasive Weed algorithm, cloud service composition, Eagle Perching algorithm, Quality of measure (Qos)

Posted Date: July 6th, 2021

DOl: https://doi.org/10.21203/rs.3.rs-583402/v1

License: (1) This work is licensed under a Creative Commons Attribution 4.0 International License. Read Full License 


\title{
A Hybrid optimization Algorithm for Enhanced Web services with QoS measures in cloud computing.
}

\author{
${ }^{1 *}$ L.Thenmozhi, ${ }^{2}$ Dr.N.Chandrakala \\ ${ }^{1}$ Assistant Professor, Department of Computer Science, MGR College, Hosur, India. \\ ${ }^{2} \mathrm{HOD}$, Department of Computer Science, SSM College of Arts \& science, \\ komarapalayam, India.
}

Corresponding Author Mail ID: Thenmozhiphd08@yahoo.com

\begin{abstract}
:-
In the Environment of Big-Data analytics in world-wide, the cloud web-services were deployed in internet and Intranet domains. Moreover cloud computing possess the privileges and acquired rapid development, faces the trust complexities, privacy concepts and security issues which allows to implement the QoS measures in the optimization techniques in the web services selection. The study focusses on selection of component services and employing the efficient algorithm with end to end Quality of measures. The Data diversification and the service characteristics would decline the accuracy level of the measures. In this study a novel Qos measure web-services algorithm implemented the weight attributes and the subjective attributes. This study employs the novel hybrid-optimization algorithm in gaining the privileges of the search randomised-attributes and the implementation of IWO-invasive-weed algorithm. This study also focusses on the Calculation of Quality-of-service measures on the weights of the web-services attributes. Many researches have placed the Implementation of nature inspired concept for the optimization complexities in Big Data and thus employing Eagle-Perching Algorithm in the efficiency enhancement of cloud webservices. The evolution of BES- Bald EagleSearch were utilized as the nature inspired approach would drive as the efficient technique for optimisation issues which imitates the bald-eagles behaviour. The results have been demonstrated the comparison of the performance metrics with the existing approaches to evaluate the proposed methodology.
\end{abstract}

Keywords: - Hybrid Invasive Weed algorithm, cloud service composition, Eagle Perching algorithm, Quality of measure (Qos)

\section{Introduction}

The Significance of Cloud computing has been predominant paradigm to assist IT service and product applications and medium to small scale businesses .The privileges of cloud computing are multi-fold such as indulging the share and the optimized usage of Unrestricted flexibility and Scalability, IT cum resources, greater automation level, services access range and decrement in the software cost and computer costs. Moreover this cloud computing possess the privileges and has rapid development, the part also faces the trust complexities, privacy concepts and Security issues which allows to implement the Qos measures in the optimization techniques .[1] Generally Cloud computing paradigm involves the various kinds of services such as PaaS-Platform as a-service, IaaS-Infrastructure as a-service and SaasSoftware as a-service. The Third-Party cloud computing providers involve in the maintenance of the services and the computation of the services infrastructure rather than organisation full-fledged participation in the investment of large amount of money for this purposes. Hence due to the privilege of the Organisations, in carrying out the cloud computing technology, cloud services attain the proliferation level in the market in the recent decade.

There is an utmost complexity in the selection of the optimal services in cloud computing 
which convinces the necessities of the users. The consistence of the composite service is defined by the services-repository wherein each separate cloud services is fetched on the basis of the distinct metrics values .The objective of cloud service-composition is to satisfy the functionality requirements of the business-logics. This composition of Composite cloud service has been specialized by the non-functional components referred as Quality of Service parameters.[2] This Qos Parameter would aid the users to evaluate the Composition in the Quality of service. In this survey, the novel approach for optimizing the cloud service selection by the implementation of Eagle Search algorithm and Invasive Weed optimization algorithm ensured with Quality of measures. [3]The study focusses on selection of component services and employing the efficient algorithm with end to end Quality of measure, The Qos measures have been presented in the dynamic web services composition and the Qos Optimization in composite service. These approaches are too sensitive to the setting parameters and easily placed in the premature convergence. In the Other side, the Metaheuristic algorithms would be the proficient approach for non-linear optimization complexities.

In the recent decades, among the researchers the computation based on the nature inspired ones have been the significant and attentive concept for the modelling of artificialcomputation system.[4] Nature is driven as the significant source of innovative ideas, concepts and the thoughtful mechanisms in the cloud computing technologies and also in resolving the mathematical conflicts. The evolution of BES- Bald Eagle-Search were utilized as the nature inspired approach would drive as the efficient technique for optimisation issues which imitates the baldeagles behaviour while in the search of prey.[5] The study on the proficient Quality of Service data would have a place in SOCService Oriented Computing. The measure on Quality of measure ought to be the co-activity of the preferences, services and users set .[6] The Data diversification and the service characteristics would decline the accuracy level of the measures. In this Survey a novel Qos measure web-services algorithm implemented the weight attributes and the subjective attributes.[7]

The major contribution involved in the study were:

- Implementation of the optimization techniques with respect to cloud web services in Big Data in an efficient way.

- Evolution and implementation of global Hybrid Invasive weed algorithm for the input of preprocessed dataset and performing the optimized system of web services.

- Calculation of Quality-of-service measures on the weights of the webservices attributes.

- Implementation of nature inspired concept for the optimization complexities in Big Data and thus employing Eagle-Perching Algorithm in the efficiency enhancement of cloud web-services.

- Comparison of the performance metrics results with the existing approaches to evaluate the proposed methodology.

\section{Related Works}

The following section deals with the literature review of the optimization in big data, invasive weed algorithm researches and eagle perching algorithm.

\subsection{Big Data optimization}

In this study, it explored the several ways in the integration in the analytics of Big Data along with the optimization approach of big data Network This paper explores various means of integrating Big Data analytics with network optimization. This optimization would have the goal in the enhancement of quality of the user. For this study, BDD- Big Data Driven optimization framework prevailing in the mobile networks have been implemented in the study. In this research [8] presented the current cross disciplinary attempts in the Big-Data science. This article also encompasses the design models from the 
varied Signal processing related tasks of data analytics which includes DL-dictionarylearning, subspace- clustering, CScompressive-sampling and in PCA-principal component-analysis.

In this article [9] demonstrates the present enhancements in optimization field by modernized data lens. This article also outlines the vital challenges and the characterization of the optimization in Big Data-driven platform. This approach also incorporates the mathematical-programming and ML-machine learning processes for benefits of decision making phenomena. It also highlights the capable research choices. In this article [10] proposed the algorithm for the purpose of multi stage processing techniques prevailing in Big Data analytics. This would cache the interface data sets and to determine the data sets which can be reutilized.

Likewise, [11] demonstrated the 3 algorithms for the process of parameter-tuning and to transmit the scheduling to the transfer throughput level at the maximum range. This resultant inferences would in turn enhance the transfer throughput level to ten times as such. This study is prevalent in broader areanetworks. In this article, [12] addressed the methodologies of decision making process which permits the non-programmers to assess and in generation of the system-operation substitutes and in selecting the one on the basis of given criteria. This study would be applicable to rectify the optimization problem.

Similarly in this article [13] determined the evolving algorithm and in the improvisation techniques to handle the sparseness conflicts and the higher-dimensionality complexities in Big-Data optimization. This study utilizes the genetic-operators to extend the range of search processes and to present the diversification in Big Data. This study [14] illustrated the decomposition-framework in optimization techniques parallel. The optimization processes is carried out for the summation of individual non-smooth convex function and the differentiable function as well. The framework found to be strongly flexible and it constitutes the Gauss Seidel scheme and Jacobi Scheme also.
In this article [15] establishes the BSUMBlock Successive Upper-Bound Minimization which founds to be the strong algorithm for the optimization of Big Data. The major advantage of the algorithmicframework is that it possess flexibility of framework and the convergence-guarantee of the algorithm. In this study [16] implemented the adaptive-mutation operators which aims to improvise the standardized-NSGA third algorithm performance. This mutationoperators approach has been analysed upon 2 cross-over NSGA Third algorithmic operators which covers the SI-single-point cross-over, SBX-simulated-binary cross-over and the UC-Uniform cross-over.

\subsection{Invasive Weed algorithm:}

This research paper [17] illustrates the SCOS - optimal selection problem and Quality of service-aware service composition problem . These problems have been outlined as the optimization-problem .Hence the modified approach discrete IWA-invasive-weed algorithm were implemented and considered as the new-methodology for the rectification of the optimal selection problem NP based issue in the cloud manufacturing frame. The functionality of the optimization approach is analysed by various multi-dimensional benchmark functions. The resultant information which is implemented by the proposed approach exhibits the high level performance in comparison with the all-other conventional approaches .The paper majorly focuses on the address of the IWO algorithm which dwells as the robust and effective algorithm for optimization.

The objective of the paper [18] stated the new approach known as Hybrid-Algorithm where this algorithm would yields the positive attributes and reduces the negative attributes of the other two algorithms. The other two algorithms includes the grey-wolves algorithmic approach and the next one is IWO - Invasive weed algorithm .The resultant algorithm which is referred as the hybrid algorithm is Invasive-weed optimization grey-wolves optimization algorithm IWOGWO. The complication of the paper is relied in obtaining the optimal predominant 
solutions for the complexities of higher measurement and complexities of nonrestricted ones for the prevention of other local issues.

This paper [19] illustrated the novelty methodology Optimal-Fitness Aware Cloudservice Composition approach which utilizes the modified IWO algorithm - Invasive weed algorithm. This optimization algorithm deals with the multiple-Quality of Service parameter. This also further balances the connectivity restrictions and the Parameters of Quality of service. Here in this paper, the performance of the methodology were assessed upon real-world cloud services Information set. Hence the focus of the paper relied on to determine the better most optimalfitness aware composition of the same cloud service. This research [20] presents the fast relevant classification optimization approaches . Since the significance of Data mining is found to be vital, the study suggests the four-fast growing algorithms which are classified and in compared with the other algorithms. This includes the following comparisons such as the MLP - Multi Layer Perceptron-networks implemented with IWO-Invasive weed algorithm, ICAImperialist Competitive algorithm, DEDifferential Evolution and PSO- Particle swarm optimization algorithm. These phenomenal classifications were performed on the data base of WBS- Wisconsin Breast Cancer. On the inferences, the algorithm are further categorized with the another kinds of GA - Genetic algorithm classifiers, which exhibited the accuracy of the classifiers and the speed-level of the categorized classifiers.

The research [21] presents the novelty optimization approach as the hybrid algorithm which retrievs the random properties of the IWO-Invasive weed optimization methodology and the chaoticsearch. The Proposed functionality of the optimization method were determined by the benchmark functions of multi-dimensional type. The resultant data proposed would exposes the higher level performance in comparison with the other conventional approaches. This paper [3] illustrated the new scheduling invasive-weed optimization algorithm for the phenomena of optimization of NFSP-new idle flow-shop-scheduling problem. This proposed methodology possess the criteria in decreasing the completion span of time at its maximum level. The resultant outcomes from the study inferences that the new idle flow-shop scheduling complexity can be rectified by the implementation of IWO-invasive weed algorithm and it qualifies with high robustness.

This algorithm in this paper [22] demonstrates the implementation of the radical dualmutation approach capable of rectifying the higher dimensional complexities. This higher dimensional issues would possess the nonhomogeneous spaces of search in consideration of the decision-variables individually. The implementation of the study on the 5 unit-system and two other various 10 unit-system exhibited the feasibility validation and efficiency validation.

The study [23] defines the service-selection complexity. This problem has been designed as the non-linear optimization methodology with the restriction constraints which led the novelty of IWO-invasive weed algorithm has been considered for study. In this research [24] provides the novel approach of Invasive weed-algorithm in Vertical Handoff for the purpose of decision-making. Hence the study of Vertical Handoff algorithm been equated with the other existing approaches of OPTG and SSF methodologies for the benefits of decision-making.

In This paper [25] defines that the methodology of Invasive weed algorithm were evaluated, launched comparison with the GA -other genetic algorithm, modelled and implemented in the studies. The comparison with the genetic algorithm-GA would be utilized to rectify personalizedurban multi criteria-quasi optimum-path problems. The inferences also illustrated the proposed study to attain the best result outcomes in terms of running time, fitness functionality and in the metrics of quality.

\subsection{Eagle perching algorithm}

In this research [26] incorporates the CSACrow-search algorithm considered as the locally involved optimizer which is of Eaglestrategy to resolve the UC problem (Unit commitment) in the smart grids technology 
systems. In this paper [5] determines the nature inspired and novel BES-Bald Eaglessearch algorithm. This BES algorithm is the optimization algorithm and also the Meta heuristic type. This algorithm would exhibits the intelligent-social strategy of the bald eagles which they the tendency in searching the prey (fish) for them.

In the research [4] implemented on the basis of the novel fresh approach eagle strategy which is an enhanced NMS-Nelder Mead simplex methodology and it is crossed with the $\mathrm{ABC}$ meta heuristic approach (Artificial bee colony). This novel proposal would require to improvise the identification of the parameter of the photovoltaic-models. [27] utilized the approach of Eagle strategy for the design-model creation of Qos-aware service composition of cloud computing. By achieving this, there established the equalization among the exploitation and the exploration of service composition. This is involved to recover the complexities of premature-convergence to that of relevant proficient point.

Likewise [28] illustrated the optimization of the Support-vector regressions parameters through evolving the BES- Bald-Eagle search algorithm with the GWO optimization algorithm (Grey-wolf optimization). The purpose of the paper is to estimate the approximation of the flow of the data traffic. In this article [29] implemented the EO approach (Eagle optimization methodology) to eliminate the Gaussian-Noise . Elimination of the noise is from the Image-data and the approach has been implemented for selecting the proper structuring-element size as well. Similarly [30] employed the Virtual MachineVM allocation policy utilizing the Eagle searching strategy involved in $\mathrm{KH}$ optimization methodology-Hybrid Krill-Herd technique. This optimization method would improvise the cloud service computing technique to all the users of Internet. This cloud-service computing also yields the connectivity of all the data-centres for resource-sharing by this Virtual-machine.

In this article [31] focusses the randomised optimization by evolving the novel two way stage hybrid methodology referred as the
Eagle-strategy. This study would reveals the combination of Fire-fly and the randomised search algorithm. This algorithm utilizes the L'evy walk approach in iterative way. The study [32] incorporates the present enhanced ES algorithm- Eagle search optimization approach) with the various distinct evolutions. This algorithm would referred as the ES-DE algorithm. This method is employed by strategy of interfacing the MATLAB software and the structuralanalysis coding (SAP2000 code). The Eagle Search-Differential evolution performance were assessed by resolving the 4 benchmark complexities. The focus of the paper also relied on the steel-frame weight minimization. In this study [33] implemented ESPSO approach for rectifying the conflicts of reactive Power-optimization and also in the power-loss elimination. The ESPSO is the enhanced particle-swarm optimizationalgorithm utilizing the Eagle-strategy method. The inferences of the results implied the proposed method to be more efficient and high level approach to the conventional approaches.

\section{Proposed Methodology}

The Primary objective of the study is obtaining the optimized selection of cloud service on the basis of implementing the Hybrid Invasive Weed algorithm and Eagle Search optimization algorithm along with Quality of Measure factors. The optimization of the cloud computing web services would be performed by the selection of Sequence set of dataset and the assessment of the Quality of measures. The data is then subjected to the optimization algorithms such as Invasive weed algorithm and Eagle search algorithm. Various web service links had been optimized. The comparison of the performance metrics of the proposed model with the conventional existing methods is performed. 


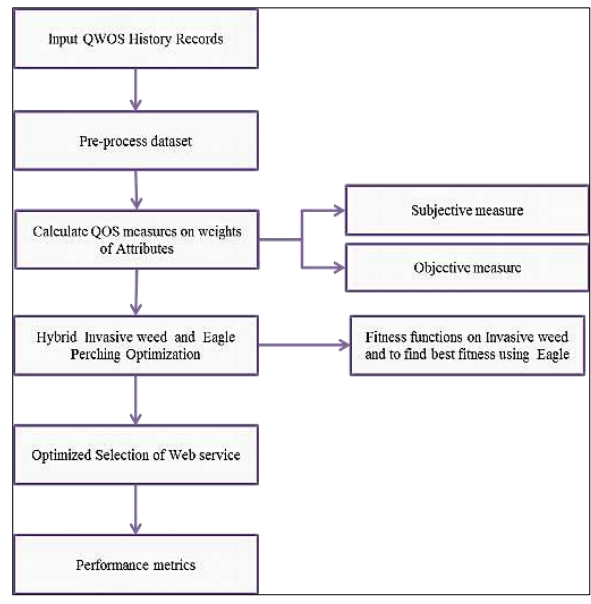

Figure 1. Proposed Overall flow

\subsection{Pre-Processing of Dataset:}

The Historical records would be fetched from the data set .This dataset describes real-world Quality of service evaluation data set results from 339 users on the count of 5,825 Web services. It has been conducted various classified larger scale Quality of service assessments of real-world web-services. This would have implemented for gaining the Quality of service datasets of the user's web services.

\subsection{QoS Measures calculation}

The data set have been subjected to the QoS calculation in terms of assessing the Throughput value and the Response time value of the selected specified web-services. This Response Time of the Web-service would be determined as time-line period between the users who transmits the request to the timeline the service-user receives the related response to the request. The Through-put is defined as the average percentage rate of the size of the message in terms of bit value which is delivered in the communication-channel each second. In order to calculate the brief demonstration of the throughput data and the response time of the selected web services by the various users, it has been chosen the 339 users from the count of 5,825 Web services. The comparison of the response time and the throughput evaluation on the distinct web services has been performed. The Prepprocessed Data set would include the Quality of services measures for the five thousand and Eight hundred and twenty five web services. These Web services would be recommended for the experimental analysis of the webservice selection.

\subsection{IWO-Invasive Weed algorithm}

This algorithm is the Meta heuristic optimization algorithm which imitates the colonization-behaviour approach of the weeds. This Algorithm is the population based dependent algorithm. [34] In this algorithm generally, the general characteristic attributes of the weed is that it increases the population as a whole or in the Particularized region which may be small or large in size. Here in the proposal, the web services count have been considered.

The 4 stepwise procedures to be followed are:

\subsubsection{Initialization Step:}

The specific number of weeds were randomly selected and scattered over the whole D dimensional-search region space. The population at the initial stage per generation will be defined as $\mathrm{X}=\left\{X_{1}, X_{2}, \ldots \ldots \ldots X_{m}\right\}$

\subsubsection{Reproduction Step:}

The number of seeds produced by the population $X_{i} \overrightarrow{\text { where }} \mathrm{i}$ belongs to $\{1,2$, $3, \ldots \mathrm{m}\}$ will have the dependency on the fitness function in accordance of worst fitness and best fitness. The population member of each were permitted for seed production within the particularized area.

\subsubsection{Spatial Distribution Step:}

The Seeds which are generated were scattered randomly upon the D dimensional space of search region. This is performed by the general random distribution of the numbers with the Variance $\sigma^{2}$ and Zero mean numbers.

$\sigma_{u}=\sigma_{\text {minm }}+\left(\frac{t_{\text {maxm }}-t}{t_{\text {maxm }}}\right)^{m \_n \_u} \cdot\left(\sigma_{\text {maximum }}-\right.$

$\left.\sigma_{\text {minimum }}\right)$

Where the $\mathrm{m} \_\mathrm{n} \_\mathrm{u}$ denotes the modulation index of non-linear form $\mathrm{t}$, $\max \mathrm{m}$ is the 
maximum count of iterations involved, and $\mathrm{t}$ represents the iteration count.

\subsubsection{Competitive exclusion step:}

This drives as the selection-procedure of Invasive weed algorithm. If there is no offspring in the growth of plants, then either it may have the chance to go extinction or would place in the world. There is a requirement factor for competition type in the plants to restrict the maximum range of plant count prevailing in population. Here in this scenario the fittest plants which include the reproduced ones and the existing plants have been considered in the colony. The step procedure of IWO algorithm were repeated unless the maximum count of the iteration numbers or the function evaluations is attained. Hence in each generation, the size of the population ought to be lesser than or equalized to the variable $p o p_{\operatorname{maxm}}$. This phenomena is referred as the competitiveexclusion.

\subsubsection{Invasive Weed Algorithm based selection of solution}

The algorithm will forward for the selection process in accordance to keep the constant value to the population size in the consecutive generations. The operation defines the survival rate of target matrix and the trial matrix to the next generation. i.e., at $n=n+$ 1. This operation of selection may be outlined as:

$$
\left\{\begin{aligned}
\vec{Y}_{i, n+1} & =\vec{U}_{i, n}, & & \text { if } f\left(\vec{V}_{i, n}\right) \leq f\left(\vec{Y}_{i, n}\right) \\
& =\vec{Y}_{i, n}, & & \text { if } f\left(\vec{V}_{i, n}\right)>f\left(\vec{Y}_{i, n}\right)
\end{aligned}\right.
$$

$\mathrm{U}, \mathrm{V}$ is two matrix ,randomly chosen index, which ensures that $\vec{U}_{i, n}$,gets at least one component from $\vec{V}_{i, n}$.

Le'vy $\sim v=n^{-\alpha},(1<\alpha \leq 3)$ here $\alpha$ is the eagle motion is essentially a random walk process with a power law step length distribution with a heavy tail.

\subsection{Eagle Search Optimization algorithm:}

The two side stage strategies of the behaviour of Eagles is considered in the approach. At first, the Eagle would be assumed that it will exhibit the Levy-walk method in entire domain. Once the Eagle find out the prey, The Eagle would alters itself to the chasing strategy. At the second side, the Chasing strategy could be presented as the intensivelocal search method mechanism utilizing the optimization-approach such as the Nelder Mead methodology. The PSO-Particle Swarm optimization algorithm have also been utilized as the effective meta-heuristic optimization algorithms, The Eagle strategy has been outlined in the pseudo code section. In this Data flow, The Fire-fly algorithms has been used as the local-search algorithm and modelled to resolve the optimization complexities globally.

\subsubsection{Weight calculation}

The Weight attribute relevant feature is denoted by $w_{i}$.

Web services $W=\left\{\mathrm{w}_{1}, \mathrm{~W}_{2}, \mathrm{~W}_{3} \ldots . . \mathrm{w}_{\mathrm{i}}\right\}$

Qos attributes $\mathrm{Q}=\left\{\mathrm{a}_{1}, \mathrm{a}_{2}, \mathrm{a}_{3}, \ldots . \mathrm{a}_{\mathrm{j}}\right\}$

While each column single QoS Parameter $Q_{j}$ denoted by $\mathrm{E}$ have two QoS parameter namely Throughput $\mathrm{Tp}$ and response time Rt

$\mathrm{q}_{\mathrm{i}, \mathrm{j}}=\mathrm{Tp}_{\mathrm{i}, \mathrm{j}} \cdot \mathrm{Rt}_{\mathrm{i}, \mathrm{j}}$

For multiply the QoS matrix here we are using strassen multiplication

$$
\mathrm{E}=\left[\begin{array}{cccc}
q_{1,1} & q_{1,2} & \ldots & q_{i, j} \\
q_{2,1} & q_{2,2} & \ldots & q_{2, j} \\
\cdot & \cdot & \cdot & \cdot \\
\cdot & \cdot & \cdot & \cdot \\
\cdot & \cdot & \cdot & \cdot \\
q_{i, 1} & q_{i, 2} & \ldots & q_{i, j}
\end{array}\right]
$$

To calculate web service relevant feature need the maximum normalized value of $Q_{j}$ column Let $\mathrm{L}$ be the array where $\mathrm{L}=\left\{l_{1}, l_{2}, l_{3}, \ldots, l_{m}\right.$ with $1 \leq m \leq \mathrm{i}$

$L(j)=\sum_{m}^{i} \mathrm{q}_{\mathrm{m}, \mathrm{j}}$

$\mathrm{M}_{\mathrm{i}, \mathrm{j}}=\frac{\mathrm{q}_{\mathrm{i}, \mathrm{j}}}{\max (\mathrm{L}(\mathrm{j}))}$

$M_{i, j}$ measures the difference from the maximum normalized value 
Different Weight contribution denoted by $\left\{\mathrm{e} 1, \mathrm{e} 2, \mathrm{e} 3, \ldots ., e_{j}\right\}$

$x_{i, j}=e_{j}\left[\frac{q_{i, j}}{\max (L(j))}\right]$

Weighted matrix denoted by E'

$\mathrm{E}^{\prime}=\left[\begin{array}{cccc}e_{1,1} & e_{1,2} & \ldots & e_{i, j} \\ e_{2,1} & e_{2,2} & \ldots & e_{2, j} \\ \cdot & \cdot & \cdot & \cdot \\ \cdot & \cdot & \cdot & \cdot \\ \cdot & \cdot & \cdot & \cdot \\ e_{i, 1} & e_{i, 2} & \ldots & e_{i, j}\end{array}\right]$

Relevant feature $(w)_{i}=\sum_{i=1}^{L} \mathrm{x}_{\mathrm{i}, \mathrm{j}}$

Hybrid Eagle Strategy with Invasive weed optimization based optimization with parallel map and reduce:

\begin{tabular}{|rl|}
\hline Invasive weed Algorithm \\
\hline 1. & Objective \\
& $f_{1}(x), \ldots, f_{t}(x)$ \\
2. & Initial guess $x^{n=0}$ \\
3. & While $\left(\left\|x^{n+1}-x^{n}\right\|>\right.$ tolerance $)$ \\
4. & Map and reduce initial values \\
5. & Random search by performing \\
& Le'vy walk \\
6. & Evaluate the objective functions \\
7. Invasive Weed Algorithm based \\
8. selection of solution \\
9. Update the current best \\
10. End if \\
11. Update $\mathrm{n}=\mathrm{n}+1$ \\
12. Calculate means and standard \\
deviations \\
13. End while \\
\hline
\end{tabular}

The random-step length factor has been taken from the distribution of Levy. The Levy-walk possess the randomised step length.

Levy $\sim \mathrm{v}=n^{-\alpha}$ where the value is $(1<\alpha$ $<=3$ ),

This has the infinite mean and infinite variance. The Eagle strategy motion is necessary as the random-walk process with step length-distribution of the power law. In this special case, the notation $\alpha=$
3 which is correspondent to Brownian motion and the notation $\alpha$ equals to 1 and it has the stochastic tunnelling characteristics.

\subsection{Map and reduce Method:}

The part illustrates the iteration sequence of Map reduce jobs. In this Map Reduce jobs count of fifty Map reduce jobs would be presented for single iteration in Map Reduce Eagle search or Invasive-weed algorithm. The resultant inference of the Map Reduce jobs relies the population which is of the updated form. This population which is of the updated one has been fetched as the input variables to the consecutive next phase of Map Reduce methodology. Master will scatters the data to ten splits of the $n$ value. (' $n$ ' equalized to 10 equivalent splits). This splits has been backed up in the distributed-file system. The number count of Map and Reduce method for three hundred and thirty-eight is one. The swarm has been spitted to the q count of populations and also the map-task of each one is overlapped with the sub-population. Such sub-population has been backed up and stored as h-key values and I pairs values. The value denotes the candidate service along with the Quality of service characteristics and key value denotes the abstract service.

The various task outcomes of the method would be transmitted as the inputs to the required Reduce functions. By the utilization of the parallel-batch processing, the reducer fetches all the data of each abstract-services. The reducer would merges all the data of iterative-sub-populations. It has been stored to the list. This whole list is then forward to the Map Reduce -MR- Eagle search /Invasive weed algorithm. This optimization algorithm would produces the output for 338 webservices individually (in terms of dominant population and the out-key). This exhibited as the perfect optimised service-composition method with robustness factor and the scalability factor.

\section{Performance Evaluation}

Comparison of the performance metrics with the existing approaches to evaluate the proposed methodology is stated in the following section. The Experimental analysis 
on the larger set of Dataset is implemented for the comparison of present proposal performance. The resultant outcome of the experiment will illustrate the efficiency of the current approach. Here in this approach, the Web service is associated to the nonfunctionality Qos-Quality of service parameter-set such as the throughput, reliability factor and the response time of the web service. This would determines the service overall performance efficiently.

\subsection{Evaluation results:}

The algorithm has been applied to the relevant benchmark of web-services challenge -WSC2009. This Dataset would give the test-sets in an efficient manner. The resultant graphical representation Consists of the web-services of the above range. The Test data set constitutes of the 4 files which includes WSDL-File which describes the registry services in the outputs and inputs. The file WSLA which stores the Quality of service such as the throughput and the response time and the service criteria values, the XML-file which stores the output and inputs of the query related to the service registry and the organized file in a hierarchical way utilizing the ontology in OWL-file[35].

\subsubsection{Comparative analysis I:}

The comparative analysis of the proposed and existing in terms of latency, response time, execution time and price, availability and success rate are depicted in this section.

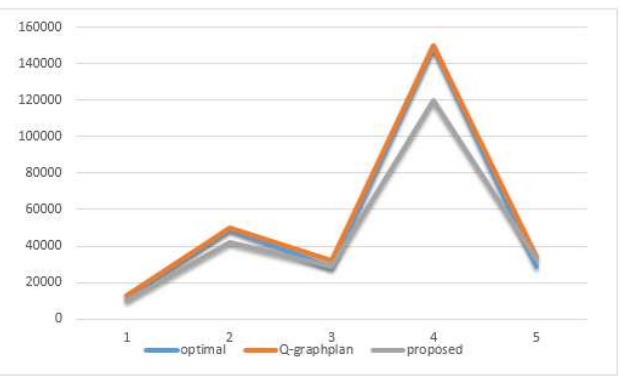

Figure 2. Latency of Optimal, Q-Graph plan and proposed result [35]

Figure 2 demonstrates the optimality concept, Q-Graph-plan's solution and proposed solution which are illustrated above. Here in this graph the latency factor is considered. The latency factor is reduced compared to the existing solutions and it shows better proposed results since the latency referred as total amount of time taken for sending the information. And it reduced for the proposed method.

Table 1: Optimality concept of Q-Graph plan and proposed result[35]

\begin{tabular}{|l|l|l|l|l|l|}
\hline \multicolumn{7}{|l|}{ Latency(ms) } \\
\hline $\begin{array}{l}\text { Optima } \\
\text { l }\end{array}$ & $\begin{array}{l}1300 \\
0\end{array}$ & $\begin{array}{l}4950 \\
0\end{array}$ & $\begin{array}{l}2800 \\
0\end{array}$ & $\begin{array}{l}15000 \\
0\end{array}$ & $\begin{array}{l}2900 \\
0\end{array}$ \\
\hline $\begin{array}{l}\text { Q- } \\
\text { graphpl }\end{array}$ & 1300 & $\begin{array}{l}5000 \\
\text { an }\end{array}$ & $\begin{array}{l}3200 \\
0\end{array}$ & $\begin{array}{l}15000 \\
0\end{array}$ & $\begin{array}{l}3400 \\
0\end{array}$ \\
\hline propose & 1000 & 4200 & 2900 & 12000 & $\begin{array}{l}3300 \\
\text { d }\end{array}$ \\
\hline
\end{tabular}

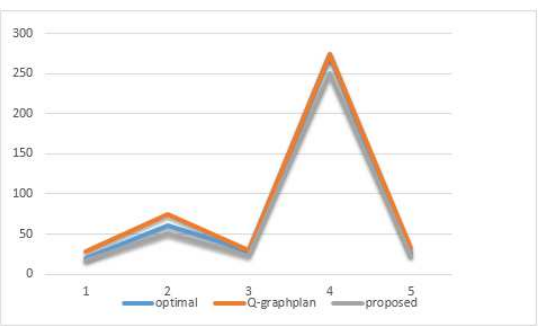

Fig 3: Execution price of Optimal, Q-graph plan and proposed result[35]

Fig.3 illustrated the execution price of the various approaches. The execution price of the proposed method and existing optimal and Q-graph plan are illustrated in fig.3 and table 2 . It shows the proposed method occupies lesser price compares with the existing methods.

Table 2: Execution time of Optimal, Qgraph plan and proposed result[35]

\begin{tabular}{|l|l|l|l|l|l|}
\hline \multicolumn{7}{|c|}{$\begin{array}{l}\text { Execution } \\
\text { Price(ms) }\end{array}$} \\
Optimal & 20 & 60 & 30 & 275 & 25 \\
\hline $\begin{array}{l}\text { Q- } \\
\text { graphpla } \\
\text { n }\end{array}$ & 28 & 75 & 30 & 275 & 32 \\
\hline proposed & 17 & 50 & 23 & 250 & 22 \\
\hline
\end{tabular}

Here in the comparison of the proposed data set of WSC 2009performance with the existing ones includes the Quality of service parameters such as execution price, response time of the web-service, latency of the data, successful-rate, reliability and the availability factor. This has been fetched from the genuine website information in the experimental 


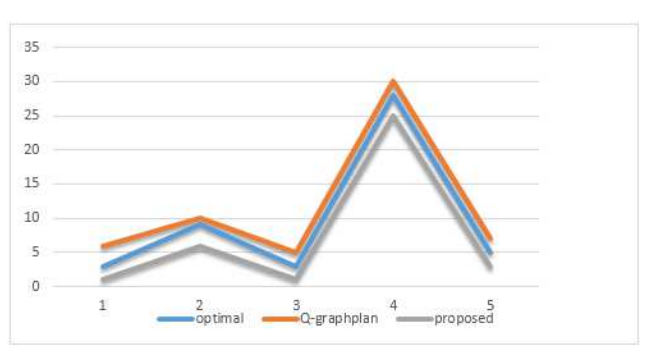

Fig.4. Reliability comparison[35]

Figure 4 and table 3 defines the reliability of the proposed and existing approaches. It shows reduced factors in reliability measurement for the proposed method compared with existing approaches depicted the better performance of the proposed method.

Table 3: Comparison of reliability[35]

\begin{tabular}{|l|l|l|l|l|l|}
\hline \multicolumn{7}{|c|}{ Reliability } \\
Optimal & 3 & 9 & 3 & 28 & 5 \\
\hline Q-graph-plan & 6 & 10 & 5 & 30 & 7 \\
\hline proposed & 1 & 6 & 1 & 25 & 3 \\
\hline
\end{tabular}

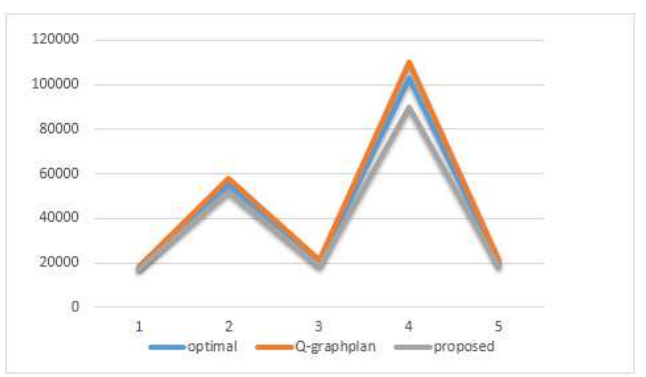

Fig.5. Response time comparison[35]

Figure 5 illustrated the various response time variations in approaches. From the table 4 and fig.5 it clearly depicted the proposed and existing response time comparison. $\mathrm{He}$ proposed method takes lesser time in execution compared with the existing method.

Table 4: Response time comparison[35]

\begin{tabular}{|l|l|l|l|l|l|}
\hline \multicolumn{9}{|c|}{$\begin{array}{l}\text { Response } \\
\text { Time(ms) }\end{array}$} \\
\hline optimal & $\begin{array}{l}180 \\
00\end{array}$ & $\begin{array}{l}5500 \\
0\end{array}$ & $\begin{array}{l}2000 \\
0\end{array}$ & $\begin{array}{l}1030 \\
00\end{array}$ & $\begin{array}{l}200 \\
00\end{array}$ \\
\hline $\begin{array}{l}\text { Q- } \\
\text { graphpla } \\
\text { n }\end{array}$ & $\begin{array}{l}180 \\
00\end{array}$ & $\begin{array}{l}5800 \\
0\end{array}$ & $\begin{array}{l}2100 \\
0\end{array}$ & $\begin{array}{l}1100 \\
00\end{array}$ & $\begin{array}{l}215 \\
00\end{array}$ \\
\hline
\end{tabular}

\begin{tabular}{|l|l|l|l|l|l|} 
proposed & 170 & 5200 & 1800 & 9000 & 180 \\
00 & 0 & 0 & 0 & 00 \\
\hline
\end{tabular}

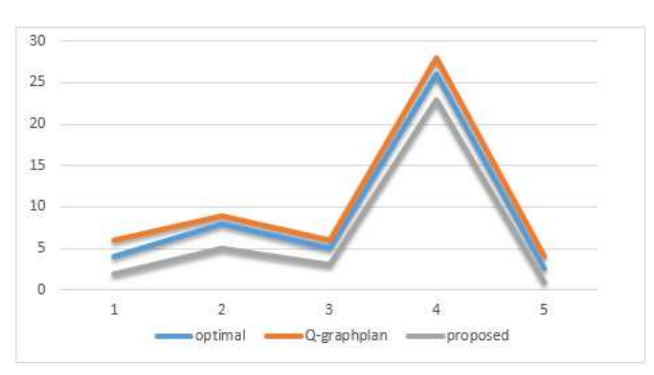

Fig.6. Availability comparison[35]

Figure 6 and table 5 presented the availability differences of the proposed and existing optimal approaches. The availability is lesser compared with existing approaches.

Table 5: Availability comparison[35]

\begin{tabular}{|l|l|l|l|l|l|}
\hline \multicolumn{7}{|c|}{ Availability } \\
\hline optimal & 4 & 8 & 5 & 26 & 2.5 \\
\hline Q-graphplan & 6 & 9 & 6 & 28 & 4 \\
\hline proposed & 2 & 5 & 3 & 23 & 1 \\
\hline
\end{tabular}

At the first stage, the optimality solution has been calculated. This optimal data values of the test-data set has been determined. The Quality of service parameter were set to detect the test data set solutions and to the same weight-measure in Q-Graph-plan.[35] The Quality of service values of Q-Graph-plan solution has been compared with the optimized values per criteria. In this graph, the red line graph denotes the Q-Graph-plan composition Quality of service values, and the blue representation of the graph denotes the optimal data values by the utilization of the single-objective techniques of optimization. The inferences of the result would implied that the representation of the Q-Graph-plan would find the solution inference where Quality of service criteria have the optimal approximation value. 


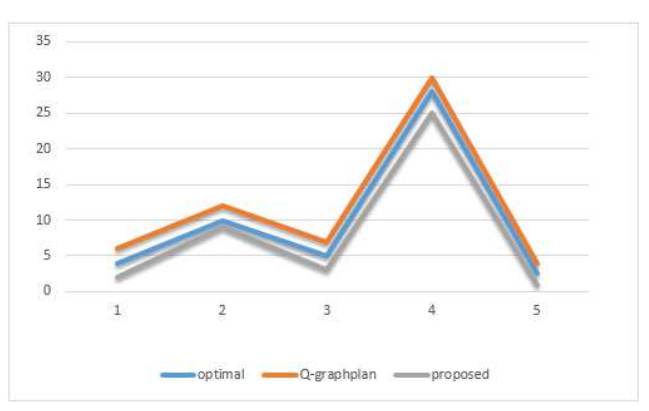

Figure 7: Success-Rate Comparisons

The Figure 7 and the Table 6 illustrated the variations in entire success rate calculations with respect to the existing approaches.

Table 6: Success rate comparisons

\begin{tabular}{|l|r|r|r|r|r|}
\hline \multicolumn{7}{|c|}{ Success } \\
optimal & 4 & 10 & 5 & 28 & 2.5 \\
\hline $\begin{array}{l}\text { Q-Graph } \\
\text { plan }\end{array}$ & 2 & 9 & 3 & 25 & 1 \\
\hline Proposed & 6 & 12 & 7 & 30 & 4 \\
\hline
\end{tabular}

\subsubsection{Comparative analysis II:}

At the second stage, the effectiveness of the algorithm is illustrated in the planning graph. The approach has evaluated the search response time on every test case several times by utilizing the mentioned two algorithms. The average response time were also calculated in the approach.

The precision methodologies of the current proposal has been compared with the serviceselection methods of the existing approaches. The service selection of the web-service were artificially presented and the resultant data of service-selection per randomised picked userrequirements.The Baseline has been obtained in synthetic-dataset and Quality of serviceselection of the web services individually.

In this approach, the Top-1 percentage, $3 \%, 5$ $\%$ and $1 \%$ were selected individually for each of the service-selection needs. The comparison of the top mentioned percentages are illustrated in below table 7 .

Table 7: Comparison of Top percentages (Precision)

\begin{tabular}{|l|l|l|l|} 
Method & Top-1\% & Top-3\% & Top-5\% \\
\hline PC\&Q & 68 & 75 & 82 \\
\hline PC\&T & 48 & 53 & 66 \\
\hline I-QP & 86 & 87 & 93 \\
\hline Integrated & 92 & 94 & 98 \\
\hline Proposed & 93 & 95 & 99 \\
\hline
\end{tabular}

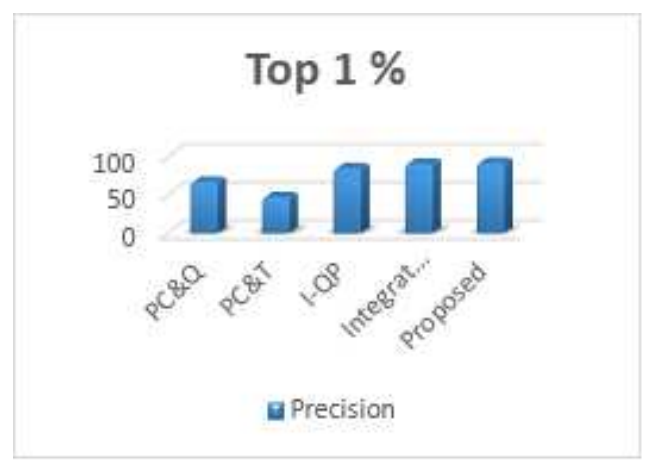

Figure 8. Precision Comparison (top $1 \%)[36]$

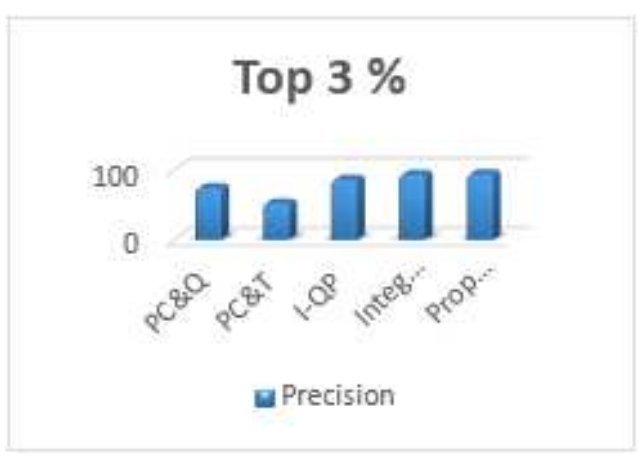

Fig. 9: Precision comparison (top 3) [36]

The service-selection mechanism per quantity of hundred requirements were conducted through PC and T, PC and Q methods, integrated methodologies in the absence of IQP Qualitative preference and the proposed methodology in separate way.[36] The results of the service-selection obtained by the various approaches would be placed in comparison with the Baseline. Hence the precision factor would be decision parameter to assess the satisfaction level of the users of several approaches. 


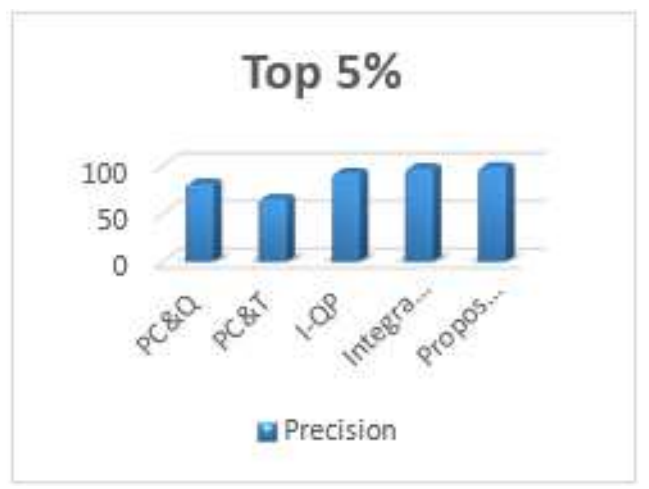

Fig.10. Precision comparison (Top 5\%)[36]

This methodology could thrive as the evidenced solution in the cloud computing application sectors of service selection of Big Data Era. The precision factor compared for the proposed and existing approaches for top 1 to $5 \%$ percentages depicted as higher values from fig.7 to fig. 10 .

\section{Comparative analysis III:}

This section deals with Reduction in Computational Complexity:

The Diagrammatic observation proven that the complexity of the computation level of Integrated-methodology is considerably low than the other previous techniques.

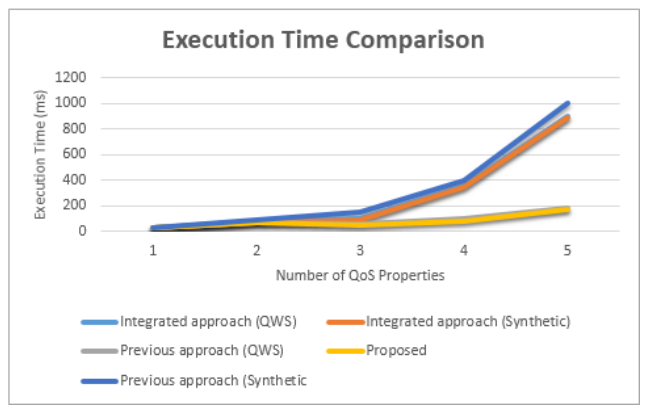

Fig. 11. Execution time comparison[36]

Fig.11, shows the execution time of various approaches illustrated above. This is due to the fact that the quality of service quantitative attributes were transmitted into the qualitative attribute approach through the other previous methods. The proposed method withstands the quantitative attributes of Quality-of- service and majorly decreases the complexities of the computation.

Hence in the summarization, the integration approach implements as the method to hold the challenge in handling the larger service data volume. This approach fits more capable for the service-selective application prevailing in Big Data domain.

\section{Conclusion}

In this Decade, the optimization technique and Quality of service-aware of the cloud web service-selection plays a significant conflict in the method of service-composition. In this focus of the paper, the issue has been designed as the non-linear optimization complexity underneath the end to end quality of service constraints. Hence it has been formulated and implemented the new Meta-heuristic search strategic approach known as Eagle Strategy (Eagle search algorithm) and effective discrete IWO-Invasive-weed algorithm to rectify this problem of computation conflicts and to encompass the optimization with the Quality Of service measures. The Experimental analysis and Theoretical assessments would present the inferences on feasibility factor, robustness measure and the efficiency of the methodology.

\section{Ethical Standards statements}

This material is the authors' own original work, which has not been previously published elsewhere. The paper is not currently being considered for publication elsewhere. The paper reflects the authors' own research and analysis in a truthful and complete manner. The paper properly credits the meaningful contributions of co-authors and co-researchers. All authors have been personally and actively involved in substantial work leading to the paper, and will take public responsibility for its content.

\section{Conflict of Interest}

I confirm that this work is original and has either not been published elsewhere, or is currently under consideration for publication elsewhere. None of the authors have any competing interests in the manuscript. 


\section{Funding}

This research work was not funded by any organization/institute/agency.

\section{Data Availability}

Not Applicable

\section{Authorship Contributions}

I Am L.Thenmozhi Hereby State That The Manuscript Title Entitled "A Hybrid optimization Algorithm for Enhanced Web services with QoS measures in cloud computing" Submitted To Wireless Personal Communications, I and my Co-author Dr. N. Chandrakala Confirm That This Work Is Original And Has Not Been Published Elsewhere, Nor Is It Currently Under Consideration For Publication Elsewhere. And I Am Assistant professor In the Department of Computer Science at MGR College, Hosur, India.

\section{REFERENCES:}

[1] Y. Pan, S. Ding, W. Fan, J. Li, and S. Yang, "Trust-enhanced cloud service selection model based on QoS analysis," PloS one, vol. 10, p. e0143448, 2015.

[2] S. Jain, M. Khandelwal, A. Katkar, and J. Nygate, "Applying big data technologies to manage QoS in an SDN," in 2016 12th International Conference on Network and Service Management (CNSM), 2016, pp. 302-306.

[3] Y. Zhou, H. Chen, and G. Zhou, "Invasive weed optimization algorithm for optimization no-idle flow shop scheduling problem," Neurocomputing, vol. 137, pp. 285 292, 2014.

[4] Q. Chen, H. Zhao, Y. Huang, J. Cao, G. Wang, Y. Sun, et al., "Effects of dietary arginine levels on growth performance, body composition, serum biochemical indices and resistance ability against ammonianitrogen stress in juvenile yellow catfish (Pelteobagrus fulvidraco),"
Animal Nutrition, vol. 2, pp. 204210, 2016.

[5] H. Alsattar, A. Zaidan, and B. Zaidan, "Novel meta-heuristic bald eagle search optimisation algorithm," Artificial Intelligence Review, vol. 53, pp. 2237-2264, 2020.

[6] L. Xin, Y. Fan, and H. Lai, "An efficient QoS preference oriented recommendation scheme for the Internet of services," in Working Conference on Virtual Enterprises, 2012, pp. 96-104.

[7] Y. Ma, S. Wang, Q. Sun, H. Zou, and F. Yang, "Web services qos measure based on subjective and objective weight," in 2013 IEEE International Conference on Services Computing, 2013, pp. 543-550.

[8] K. Slavakis, G. B. Giannakis, and G. Mateos, "Modeling and optimization for big data analytics:(statistical) learning tools for our era of data deluge," IEEE Signal Processing Magazine, vol. 31, pp. 18-31, 2014.

[9] C. Ning and F. You, "Optimization under uncertainty in the era of big data and deep learning: When machine learning meets mathematical programming," Computers \& Chemical Engineering, vol. 125, pp. 434-448, 2019.

[10] Z. Yang, D. Jia, S. Ioannidis, N. Mi, and B. Sheng, "Intermediate data caching optimization for multi-stage and parallel big data frameworks," in 2018 IEEE 11th International Conference on Cloud Computing (CLOUD), 2018, pp. 277-284.

[11] E. Arslan, B. A. Pehlivan, and T. Kosar, "Big data transfer optimization through adaptive parameter tuning," Journal of Parallel and Distributed Computing, vol. 120, pp. 89-100, 2018.

[12] A. Antonova and K. Aksyonov, "Application of a metallurgical enterprise information system for collection and analysis of big data and optimization of multi-agent resource conversion processes," 
Ekaterinburg: IT \& MathAZ, vol. 2109, p. 1, 2018.

[13] M. Bhattacharya, R. Islam, and J. Abawajy, "Evolutionary optimization: a big data perspective," Journal of network and computer applications, vol. 59, pp. 416-426, 2016.

[14] F. Facchinei, G. Scutari, and S. Sagratella, "Parallel selective algorithms for nonconvex big data optimization," IEEE Transactions on Signal Processing, vol. 63, pp. 1874-1889, 2015.

[15] M. Hong, M. Razaviyayn, Z.-Q. Luo, and J.-S. Pang, "A unified algorithmic framework for blockstructured optimization involving big data: With applications in machine learning and signal processing," IEEE Signal Processing Magazine, vol. 33, pp. 57-77, 2015.

[16] J.-H. Yi, S. Deb, J. Dong, A. H. Alavi, and G.-G. Wang, "An improved NSGA-III algorithm with adaptive mutation operator for Big Data optimization problems," Future Generation Computer Systems, vol. 88, pp. 571-585, 2018.

[17] H. Bouzary, F. F. Chen, and K. Krishnaiyer, "A modified discrete invasive weed algorithm for optimal service composition in cloud manufacturing systems," Procedia manufacturing, vol. 17, pp. 403-410, 2018.

[18] W. A. Qasim and B. A. Mitras, "AHybrid ALGORITHM BASED ON INVASIVE WEED OPTIMIZATION ALGORITHM AND GREY WOLF OPTIMIZATION ALGORITHM."

[19] C. Jatoth, G. Gangadharan, and U. Fiore, "Optimal fitness aware cloud service composition using modified invasive weed optimization," Swarm and evolutionary computation, vol. 44, pp. 1073-1091, 2019.

[20] H. Malmir, F. Farokhi, and R. Sabbaghi-Nadooshan, "Efficient Data Mining with Evolutionary Algorithms for Cloud Computing Application," International Journal of Smart Electrical Engineering, vol. 3, pp. 47-53, 2014.

[21] M. Ahmadi and H. Mojallali, "Chaotic invasive weed optimization algorithm with application to parameter estimation of chaotic systems," Chaos, Solitons \& Fractals, vol. 45, pp. 1108-1120, 2012.

[22] R. Sharma, S. K. Nayak, P. K. Rout, and K. Krishnanand, "Solution to dynamic economic dispatch problem using modified invasive weed optimisation with dual mutation strategy," International Journal of Swarm Intelligence, vol. 1, pp. 7090, 2013.

[23] K. Su, L. Ma, X. Guo, and Y. Sun, "An Efficient Discrete Invasive Weed Optimization Algorithm for Web Services Selection," JSW, vol. 9, pp. 709-715, 2014.

[24] T. Velmurugan, S. Khara, S. Nandakumar, and B. Saravanan, "Seamless Vertical Handoff using Invasive Weed Optimization (IWO) algorithm for heterogeneous wireless networks," Ain Shams Engineering Journal, vol. 7, pp. 101111, 2016.

[25] P. Pahlavani, M. R. Delavar, and A. U. Frank, "Using a modified invasive weed optimization algorithm for a personalized urban multi-criteria path optimization problem," International Journal of Applied Earth Observation and Geoinformation, vol. 18, pp. 313328, 2012.

[26] R. Habachi, A. Touil, A. Charkaoui, and A. Echchatbi, "Eagle strategy based crow search algorithm for solving unit commitment problem in smart grid system," Indonesian Journal of Electrical Engineering and Computer Science, vol. 12, pp. 17-29, 2018.

[27] S. K. Gavvala, C. Jatoth, G. Gangadharan, and R. Buyya, "QoSaware cloud service composition using eagle strategy," Future Generation Computer Systems, vol. 90, pp. 273-290, 2019. 
[28] S. Angayarkanni, R. Sivakumar, and Y. R. Rao, "Hybrid Grey Wolf: Bald Eagle search optimized support vector regression for traffic flow forecasting," Journal of Ambient Intelligence and Humanized Computing, pp. 1-12, 2020.

[29] V. Mythili and D. R. Manavalan, "Optimizing Structuring Element Using Eagle Optimization for Image Denoising: Performance Analysis," ed: IJASCSE.

[30] D. Kesavaraja and A. Shenbagavalli, "QoE enhancement in cloud virtual machine allocation using Eagle strategy of hybrid krill herd optimization," Journal of Parallel and Distributed Computing, vol. 118, pp. 267-279, 2018.

[31] X.-S. Yang and S. Deb, "Eagle strategy using Lévy walk and firefly algorithms for stochastic optimization," in Nature Inspired Cooperative Strategies for Optimization (NICSO 2010), ed: Springer, 2010, pp. 101-111.

[32] S. Talatahari, A. H. Gandomi, X.-S. Yang, and S. Deb, "Optimum design of frame structures using the eagle strategy with differential evolution," Engineering Structures, vol. 91, pp. 16-25, 2015.

[33] H. Yapıcı and N. Çetinkaya, "An improved particle swarm optimization algorithm using eagle strategy for power loss minimization," Mathematical Problems in Engineering, vol. 2017, 2017.

[34] A. Basak, D. Maity, and S. Das, "A differential invasive weed optimization algorithm for improved global numerical optimization," Applied Mathematics and Computation, vol. 219, pp. 66456668, 2013.

[35] Z. Wang, B. Cheng, W. Zhang, and J. Chen, "Q-graphplan: QoS-aware automatic service composition with the extended planning graph," IEEE Access, vol. 8, pp. 8314-8323, 2020.

[36] H. Wang, C. Yu, L. Wang, and Q. $\mathrm{Yu}$, "Effective bigdata-space service selection over trust and heterogeneous QoS preferences," IEEE Transactions on Services Computing, vol. 11, pp. 644-657, 2015. 